\title{
On the launch of the journal Information Geometry
}

\section{Shinto Eguchi ${ }^{1}$}

Published online: 19 September 2018

(c) Springer Nature Singapore Pte Ltd. 2018

It is our pleasure to publish the first issue of the journal Information Geometry in the autumn of 2018. We wish to host interesting discussions and present developments that offer insights into geometry for applications inspired by the mathematical sciences. At the same time, the crystallization of geometry from such problem solving aims as well at creating methodological foundations in geometric approaches. We believe it is important to have strong interactions between discussions of geometry and applications in the mathematical sciences.

We are grateful to the distinguished scholars who have given us congratulatory messages, namely, Professors C. R. Rao, Sir David Cox, Ole Barndorff-Nielsen, and Bradley Efron. Such encouraging good wishes from those pioneers of information geometry are highly esteemed by all of us and in particular by the younger generations. We must say that the present status of information geometry owes a profound debt to those forerunners for their invaluable contributions.

Many unsolved problems and unexplored directions remain in information geometry, however. In practice, essential questions and comments raised for information geometry are still to be fully answered and brought into the light to open new directions and frameworks.

We hope that this journal plays a fundamental role in building new perspectives and theories for information geometry. Our further hope is to provide fruitful results and deep influence in broad areas of mathematical science in the near future.

Shinto Eguchi

Editor-in-Chief

Information Geometry

Shinto Eguchi

eguchi@ism.ac.jp

1 The Institute of Statistical Mathematics, 10-3 Midori-cho, Tachikawa, Tokyo 190-8562, Japan 